\title{
Converting genetic network oscillations into somite spatial pattern
}

\author{
K. I. Mazzitello, ${ }^{1,2}$ C. M. Arizmendi, ${ }^{2}$ and H. G. E. Hentschel ${ }^{3}$ \\ ${ }^{1}$ CONICET \\ ${ }^{2}$ Facultad de Ingeniería, Universidad Nacional de Mar del Plata, Argentina \\ ${ }^{3}$ Department of Physics, Emory University, USA
}

(Dated: November 15, 2018)

\begin{abstract}
In most vertebrate species, the body axis is generated by the formation of repeated transient structures called somites. This spatial periodicity in somitogenesis has been related to the temporally sustained oscillations in certain $\mathrm{mRNAs}$ and their associated gene products in the cells forming the presomatic mesoderm. The mechanism underlying these oscillations have been identified as due to the delays involved in the synthesis of mRNA and translation into protein molecules [J. Lewis, Current Biol. 13, 1398 (2003)]. In addition, in the zebrafish embryo intercellular Notch signalling couples these oscillators and a longitudinal positional information signal in the form of an Fgf8 gradient exists that could be used to transform these coupled temporal oscillations into the observed spatial periodicity of somites. Here we consider a simple model based on this known biology and study its consequences for somitogenesis. Comparison is made with the known properties of somite formation in the zebrafish embryo. We also study the effects of localized Fgf8 perturbations on somite patterning.

PACS numbers: 05.40.-a, 05.60.-k, 73.63.-b
\end{abstract}

\section{INTRODUCTION}

Somites are transient structures that form a periodic growing pattern starting from the head (anterior) and extending to the tail (posterior) of a developing vertebrate embryo which ultimately give rise to both the segmented vertebral column and to the musculature. Formation of somites is a rhythmic process characteristic of the species at given temperature. For instance, in the chicken embryo one pair of somites is formed every $90 \mathrm{~min}$ at 37 ${ }^{\circ} \mathrm{C}$ and in the zebrafish one pair is formed roughly every $30 \mathrm{~min}$ at $28^{\circ} \mathrm{C}$. The origin of these intracellular oscillations have been identified as due to time delays in the transcription and translation of her 1 and her 7 genes in the case of zebrafish and the hes 7 gene in the case of mouse. The total number of somites produced is conserved within a given species typically somewhere between 50 to 70 pairs of somites form on each side of the anterior-posterior (AP) axis, but can vary dramatically between species, thus the zebrafish develops 30 pairs of somites. As new somites bud from the anterior end of the presomatic mesoderm (PSM) that extends back to the primitive streak and tail bud, new cells are added at the posterior end by cell division from the tail bud keeping the size of the PSM constant as new somites are generated .

Somitogenesis is one of the best studied process of pattern formation in the developing embryo, and a number of models have been proposed to address the mechanisms underlying the generation of such periodic patterns [2, 4, [5, 7, 8, 9]: the Clock and Wavefront model proposed by Coke and Zeeman in 1976 is perhaps best known, but others include Meinhardt's Reaction Diffusion model; and Stern's Cell Cycle model. All of them suppose that oscillations of genes and gene products oc- cur in the cells of the PSM from which the somites derive (Fig. 11). The Clock and Wavefront model postulates the existence of a longitudinal positional information gradient down the axis of vertebrate embryos. This gradient interacts with the cellular oscillator stopping the oscillations and producing a rapid change in locomotory and adhesive behavior of cells when they form somites 3, 11. The oscillation in the PSM is the somite clock, and the moving interfaces at the anterior end of the PSM where the positional information reaches a critical value is called the wavefront. It is the interaction beween the clock phase and the positional information that controls somitogenesis. Here we suppose the positional information is supplied by the concentration gradient of the gene product Fgf8 released at the tail bud of the embryo and creating a linear gradient of morphogen along the AP axis [2, 3]. Biologically the signal created by the Fgf8 concentration interacts with the clock phase (the intracellular concentration of a gene protein) leading to cell differentiation and somite formation. At low Fgf8 concentration, cell arrangement becomes more compact and the epithelialization process underlying somite formation begins.

Meinhardt's Reaction Diffusion model also incorporates the idea of an oscillatory mechanism in the PSM cells producing an alteration between cell states as in the Clock and Wavefront model. One of the main aims of this model is to account for somite structure itself which at even early stages after formation shows heterogeneities between the anterior and posterior portion of an individual somite. To account for this structure the reaction diffusion model postulates that cells can be in one of two possible states: anterior $a$ and posterior $p$ and switch from one state to another until they reach a stable state in the presence of a morphogen gradient forming a spatial 
pattern such as aaappaaapp..., where a repeated segment aaapp constitutes a somite.

Finally Stern's Cell Cycle model assumes that the oscillations in the cells arranged along the AP axis are in synchrony with each other, but cells in the anterior PSM are further advanced in phase than cells in the posterior PSM. Segmentation is hypothesised to occur when cells reach a certain time window in their cell cycle. The most important point raised by this model is the question of how cells are synchronized biologically. It appears that the answer to this question lies in the deltaC gene that codes for the Notch ligand at the heart of the intercellular Notch signalling pathway which when disrupted destroys cell cycle synchrony [1]. All these models raise important questions, but recent experiments on how Fgf8 signalling controls somite boundary position and regulates the segmentation clock [11] appears most in accord with the clock and wavefront model.

Based in the Clock and Wavefront model combined with key ingredients of the known biology, we have therefore constructed a deterministic one dimensional model and compare the resulting spatial patterning with the somites for zebrafish embryo. We use in our model the recent proposal for the mechanism producing the intracellular oscillations as due to delays in the synthesis of mRNA focussing specifically on the zebrafish genes her 1 and $h e r 7$ and their gene products the protein molecules Her1 and Her7 [1].

The paper is outlined as follows: Section $\amalg$ is devoted to the description of the proposed model and contains the results derived from it. The transcription in the synthesis of mRNA is essentially a noisy process and is considered for haploid cells in the section III. In section IV. we study the effect of a local perturbation of the Fgf8 wavefront on the spatial pattern of somites. Finally a conclusion and discussion is presented in $\mathrm{V}$.

\section{DETERMINISTIC ONE DIMENSIONAL MODEL}

The model consists initially of a linear array of $N_{\text {start }}$ cells that represents the starting size of the PSM with intracellular oscillators driven by the her 1 and her 7 genes and their associated gene product proteins. The oscillations between nearest-neighbor cells are coupled and synchronized via Notch signalling [13, 14]. The cell-cell communication produced by means of the deltaC gene product Notch crossing the cell membrane wall.

In each cell in the array the gene mRNA concentrations $m_{k}$ for the genes $k \equiv$ her 1 , her 7 , deltaC and their associated gene products the protein concentrations $p_{k}$ obey the sets of coupled kinetic equations (see [1]):

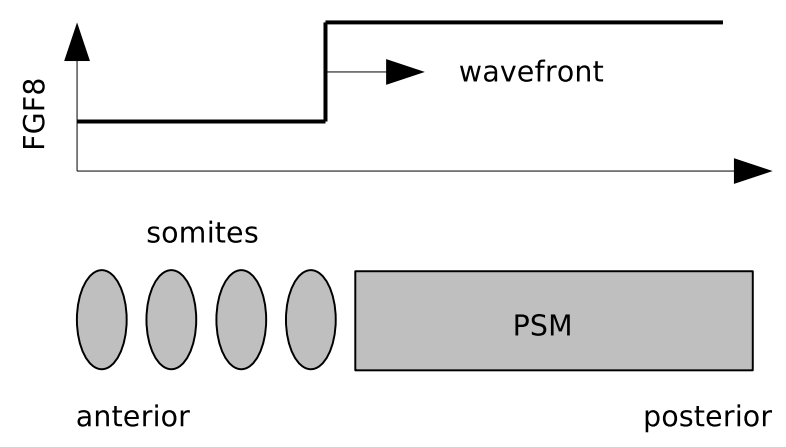

FIG. 1: An schematic illustration of somite formation within the Clock and Wavefront model. Gene and gene product concentrations in the cells of PSM experience temporal oscillations. In the top part of the diagram, the positional information wavefront is illustrated together with the position of the determination front which we assume represents the position at which the Fgf8 concentration gradient falls below a critical value. The somites form in an anterior to posterior order as the wavefront advances from head to tail.

$$
\begin{aligned}
\frac{d p_{k}}{d t}(i, t)= & a_{k} m_{k}\left(i, t-T_{p_{k}}\right)-b_{k} p_{k}(i, t) \\
\frac{d m_{k}}{d t}(i, t)= & \frac{1}{n n} \sum_{i^{\prime}=1}^{n n} f_{k}\left(p_{h e r 1}\left(i, t-T_{m_{k}}\right), p_{h e r}\left(i, t-T_{m_{k}}\right)\right. \\
& \left.p_{\text {deltaC }}\left(i^{\prime}, t-T_{m_{k}}\right)\right)-c_{k} m_{k}(i, t)
\end{aligned}
$$

Here the symbol $i$ denotes the cell position in the linear array; $i^{\prime}$ goes from 1 to $n n$ the number of near neighbor cells of $i$ ( $n n$ can be 1 if $i$ is at an end or otherwise 2$) ; T_{m_{k}}$ is the delay time from initiation of transcription to export of the mature mRNA $m_{k}$ into the cytosol; $T_{p_{k}}$ is the delay between the initiation of translation and the emergence of the complete protein molecule $p_{k} ; a_{k}$ represents the protein $p_{k}$ synthesis rate per mRNA molecule; $b_{k}$ is the rate of protein $p_{k}$ degradation; $c_{k}$ is the rate of mRNA $m_{k}$ degradation; while the function $f_{k}$ represents the rate of production of new mRNA molecules $m_{k}$ that is given by the Michaelis-Menten kinetics [1]:

$$
\begin{aligned}
& f_{k}\left(p_{\text {her } 1}\left(i, t^{\prime}\right), p_{\text {her } 7}\left(i, t^{\prime}\right), p_{\text {deltaC }}\left(i^{\prime}, t^{\prime}\right)\right)=K_{k}\left\{r 0_{k}\right. \\
& +r d_{k} \frac{\phi_{\text {deltaC }}\left(i^{\prime}, t^{\prime}\right)}{1+\phi_{\text {deltaC }}\left(i^{\prime}, t^{\prime}\right)}+r h_{k} \frac{1}{1+\phi_{\text {her } 1}\left(i, t^{\prime}\right) \phi_{\text {her } 7}\left(i, t^{\prime}\right)} \\
& \left.+r h d_{k} \frac{\phi_{\text {deltaC }}\left(i^{\prime}, t^{\prime}\right)}{1+\phi_{\text {deltaC }}\left(i^{\prime}, t^{\prime}\right)} \frac{1}{1+\phi_{\text {her } 1}\left(i, t^{\prime}\right) \phi_{\text {her } 7}\left(i, t^{\prime}\right)}\right\}
\end{aligned}
$$

where we used the notation $t^{\prime}=t-T_{m_{k}}$; and $\phi_{k}(i, t)=p_{k}(i, t) / p_{k}^{c r i t}$. The parameters $r 0_{k}, r d_{k}, r h_{k}$, and $r h d_{k}$ that add up to 1 , represent the relative weights of $k$ transcription that is unregulated, regulated by 
deltaC protein alone, regulated by her protein alone, and regulated by her and deltaC proteins, respectively. $p_{k}^{\text {crit }}$ is the critical number of molecules of Her1 or Her7 protein per cell, for inhibition of transcription if $k$ is her 1 or her 7 respectively, while it represents the critical number of Notch molecules required for activation if $k$ is deltaC. The mRNA concentrations for the kind different of genes decrease when the $k$ protein concentration are larger than their critical values $p_{k}^{\text {crit }}$ if $k$ is Her1 or Her7 or when the deltaC protein Notch concentration is below its critical value $p_{\text {deltaC }}^{\text {crit }}$. The concentrations of intercellular signalling molecules in the cells are coupled between the nearest-neighbour $n n$ cells by means of the function $f_{k}$ through the deltaC protein Notch concentration of their neighboring cells $\left.p_{\text {deltaC }}\left(i^{\prime}, t-T_{m_{k}}\right)\right)$, as it can be seen in the Eq. (2).

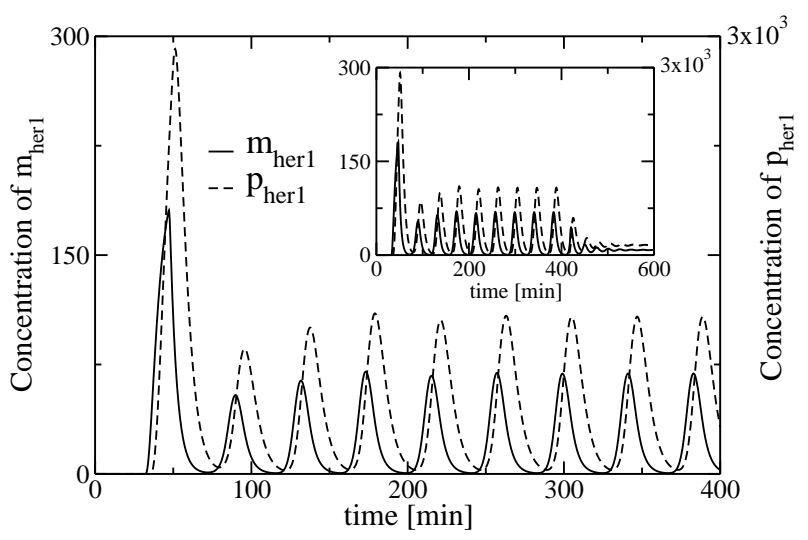

FIG. 2: Concentrations of mRNA molecules of gene her 1 and its associated gene product protein Her1 as a function of time for any cell of the linear array. Sustained oscillations of both concentrations are obtained for the following values of parameters involve in Eqs. (1) and (2): $a_{k}=4.5, b_{k}=0.23, c_{k}=0.23, K_{k}=33, r 0_{k}=r d_{k}=0$, with $k=$ her 1, her 7, deltaC $, r h_{\text {her } 1, \text { her } 7}=0, r h_{\text {deltaC }}=$ $1, r h d_{\text {her } 1, \text { her } 7}=1, r h d_{\text {her } 1, \text { her } 7}=1, r h d_{\text {deltaC }}=0$, $p_{\text {her } 1, \text { her } 7}^{\text {crit }}=40, p_{\text {delta }}^{\text {crit }}=1000, T_{m_{\text {her } 1}}=12, T_{m_{\text {her } 7}}=7.1$, $T_{m_{\text {deltaC }}}=16, T_{p_{\text {her } 1}}=2.8, T_{p_{\text {her } 7}}=1.7, T_{p_{\text {deltaC }}}=20 \mathrm{~min}$. (Inset) The oscillations are stopped when the corresponding cell is reached by the somitogenesis wavefront. The concentrations of protein and mRNA molecules of genes her 7 and deltaC have a similar behavior as the concentrations of $m_{\text {her } 1}$ and $p_{h e r 1}$ shown in this figure.

The concentrations of both protein and mRNA molecules, $p_{k}$ and $m_{k}$, are set equal to zero initially in all $N_{\text {start }}$ cells of the linear array. The temporal evolution of $p_{k}$ and $m_{k}$ are calculated with Eqs. (11). Sustained oscillations of mRNA and proteins are obtained for parameters and time delays $T_{m_{k}}$ and $T_{p_{k}}$ close to the zebrafish estimated ones as it is shown in Fig. 2] [1]. The mRNA and protein oscillations can occur without interactions between neighbor cells, because the delays that take part in feedback loops are intracellular. The

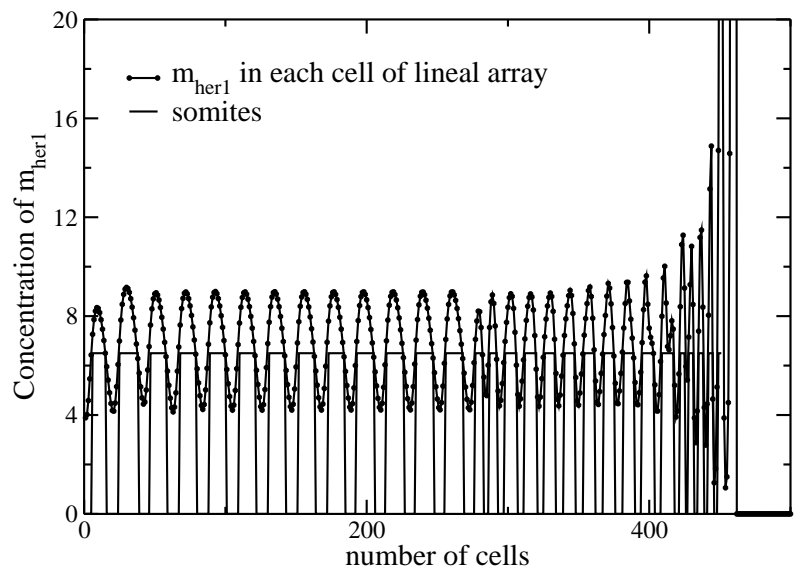

FIG. 3: Concentration of mRNA molecules of gene her 1 as a function of cell number in the somites. The concentrations were calculated using Eqs. (11) and (2) and using the same parameter values as in the Fig. 2 (see caption). The concentration of $m_{\text {her } 1}$ belonging to cells that were reached by the wavefront were frozen. In our simulation the wavefront advances at a speed of one cell every two minutes. We see the simulation at time $t=900$ minutes at the end of somitogenesis in the zebrafish. At $t=900$ minutes 29 somites have formed. All the other concentrations of protein and mRNA molecules in the cells (namely $p_{k}$ with $\mathrm{k}=$ Her1, Her7, Notch and $m_{k}$ with $k=$ her 7 , deltaC) have the same qualitative behaviour as the concentration of $m_{\text {her } 1}$.

interactions through $f_{k}$ connecting neighbor cells are, however, crucial as they result in synchronous in phase oscillations in the linear array representing the PSM as a whole to occur.

We suppose at time $t=0$ all the cells in the starting PSM to be oscillating in phase, and therefore we begin with the linear array of $N_{\text {start }}$ cells and integrate them for the time necessary to obtain coherent synchronous oscillations. In this paper $N_{\text {start }}=280$, and in its synchronous oscillating state it represents the time $t=0$ in our model. The growth of the PSM is simulated in our model by the adding of new cells to the initial group of cells at one end of the linear array. This growth velocity $v_{\text {growth }}$ is a variable parameter in our model, and we chose to add cells at a rate of one cell every 5 minutes in agreement with measured growth rates [10]. Thus, it generates sequentially along the antero-posterior axis the necessary cells for forming the future segments of the embryo: the somites that are created at the anterior portion of the PSM, see Fig. 1. In our simulations once the initial PSM has been obtained as a group of cells oscillating in phase, the caudal motion of the wavefront starts. Again the wavefront velocity $v_{\text {wave }}$ is a parameter in the model that controls the rate at which cells reached by the wavefront stop oscillating and thus freezing the gene and gene product concentrations at their values when they meet the wavefront (see inset of Fig. 2). The observed spa- 
tial shift in the phases of different cells is thus obtained by the wavefront catching different phases when passing through different cells, a biological implementation of the clock and wavefront model.

What value should we choose for $v_{\text {wave }}$ ? The simplest argument would suggest $v_{\text {wave }}=v_{\text {growth }}$. This supposes that the FGF creates a time indepedent distribution on a fast timescale compared to growth, and therefore the wavefront is simply reading off a critical value of the FGF concentration at which the intracellular oscillator is frozen. If we chose this value, however, we would not create around 30 somites in 900 minutes as we would expect [10]. Indeed, it is important to note that the regularity of the somite pattern and the size of somites is very sensitive to changes in $N_{\text {start }}$ and $v_{\text {wave. }}$ For example, if $N_{\text {start }}=20$ and $v_{\text {wave }}=v_{\text {growth }} \approx 5 \mathrm{~min}$ per cell, the number of somites is about 10 for 900 minutes. Here we report instead on our simulations with $v_{\text {wave }}=2$ minutes per cell. Then when the simulation finishes in the time $t=900$ minutes we find that 29 somite pairs have been created. A wavefront advancing at a rate of 1 cell $/ 2$ minutes creates a spatial wavelength $\lambda=T_{\text {cell }} v_{\text {wave }} \approx 15$ cells in length. What is the process of cell differentiation after the cell meets the wavefront now depends on the biology. Here to be specific we have assumed that when the $m_{h e r 1}$ level is above its average value a somite is formed. Averages of the concentrations of proteins and mRNA of the frozen cells are calculated at this final time. With this choice a somite is roughly $6-7$ cells in length. On the other hand the neighbor cells that have protein and mRNA concentrations lower than average form the space between somites. With this choice the remaining $8-9$ cells form an intersomite gap. Of course, it is also possible to interpret the simulation as forming somites of length 15 cells with a differentiated structure between the anterior and posterior portions of each somite. Now as the simulation proceeds the PSM reduces in size. Indeed, for the parameters chosen here $N_{\text {start }}=280$ cells, but $N_{\text {end }}=10$ cells, because while 180 cells are added in the 900 minutes of the simulation at the posterior end of the PSM, a total of 450 cells differentiate into new somites, It is not surprising therefore that phase coherence is lost towards the end of somitogenesis, and the spatial array of somites obtained, though approximately periodic, decrease in size towards the tail (Fig. 3).

\section{STOCHASTIC MODEL}

Our simulations above assumed that no noise was present in the cells during transcription and translation, but as Lewis has pointed out, the transcription step involved in protein synthesis is essentially a stochastic process because of the small number of molecules involved in the cell [15]: A DNA molecule can randomly have a Her1, Her7 or Delta Notch dimer bound to its regula- tory site. When such a dimer does bind, transcription is forbidden; whereas if no protein is bound at a regulation site of the DNA molecule, transcription occurs at the possible maximal rate.

The stochastic model for haploid cells is then constructed by taking the deterministic one dimensional model and considering the possible states (bound and dissociated) of all regulatory sites of the her 1 , her 7 and deltaC genes as stochastic variables [1]. A DNA regulatory site can have a Her1, Her7 or Delta Notch dimer bound or not (i. e. either transcription forbidden or maximally free of repression). A gene regulatory site then makes stochastic transitions between the bound and unbound states with a certain probability in any time interval.

The protein and mRNA concentrations are given by the Eqs. (11) as in the deterministic case, but the function $f_{k}$ now describe a stochastic process and are therefore modified to:

$$
\begin{aligned}
& f_{k}\left(p_{\text {her } 1}\left(i, t^{\prime}\right), p_{\text {her } 7}\left(i, t^{\prime}\right), p_{\text {delta }}\left(i^{\prime}, t^{\prime}\right)\right)=K_{k}\left\{r 0_{k}\right. \\
& +r d_{k}\left[1-\xi_{k}^{(D)}\left(i^{\prime}, t^{\prime}\right)\right]+r h_{k} \xi_{k}^{(H)} k\left(i, t^{\prime}\right) \\
& \left.+r h d_{k} \eta_{k}^{(H)}\left(i, t^{\prime}\right)\left[1-\eta_{k}^{(D)}\left(i^{\prime}, t^{\prime}\right)\right]\right\}
\end{aligned}
$$

where the parameters $r 0_{k}, r d_{k}, r h_{k}$, and $r h d_{k}$ were defined previously, below of Eq. (2). The functions $\xi_{k}^{(D)}$, $\xi_{k}^{(H)}, \eta_{k}^{(H)}$ and $\eta_{k}^{(D)}$, with $k=$ her 1 , her 7 , deltaC, are random variables $(r v)$ taking a value 1 when no protein is bound at the regulatory site and 0 when it is bound, with probabilities that depend on protein concentrations in the cell $i$ and in its neighbor cell $i^{\prime}$, at time $t^{\prime}=t-T_{m_{k}}$ when synthesis of $m_{k}(t)$ begins. These probabilities at a given time $t$ are thus conditioned by the values the random variables take at an earlier time $t^{\prime}$. Specifically the probability that any random variable $(r v)$ takes value 1 at the time $t+\Delta t$ depends on the state of the appropriate regulatory site at time $t$ (bound or unbound).

$$
\begin{aligned}
p_{10}^{r v}(t+\Delta t)= & 1-P^{r v}(t+\Delta t) \mid\left(P^{r v}(t)=0\right), \text { if regulatory } \\
& \text { site is bound at time } t \\
p_{11}^{r v}(t+\Delta t)= & P^{r v}(t+\Delta t) \mid\left(P^{r v}(t)=1\right), \text { if regulatory } \\
& \text { site is unbound at time } t
\end{aligned}
$$

In Eq. $4 p_{\alpha \beta}^{r v}$ represents the probability that any random variable $r v$ has value $\alpha$ at $t+\Delta t$ if it had value $\beta$ at $t$ (rv can be $\xi_{k}^{(D)}, \xi_{k}^{(H)}, \eta_{k}^{(H)}$ or $\eta_{k}^{(D)}$, and $\alpha=1, \beta=0,1$ ); while $P^{r v}(t)$ is the probability that the regulatory site is free of repression. This probability obeys the master equation

$$
d P^{r v} / d t=k_{o f f}^{r v}\left(1-P^{r v}\right)-k_{o n}^{r v} P^{r v} .
$$

These kinetic coefficients are discussed in [1] (Supplemental Data), where $k_{o n}^{r v}$ the rate constant for protein binding 
is a MIchaelis-Menten function of the protein concentrations, while $k_{o f f}^{r v}$ is the rate constant for dissociation of the repressor proteins from their regulatory DNA binding sites (typically biologically $k_{o f f}^{r v} \approx 1 \mathrm{~min}^{-1}$ implying a mean lifetime of about 1 min for the repressor bound state).

Solving the above differential equation results in the forms $P^{r v}(t+\Delta t)=u^{r v}(1-\exp [-v \Delta t])+$ $P^{r v}(t) \exp \left[-v^{r v} \Delta t\right]$, where $v^{r v}=\left(k_{o f f}^{r v}+k_{o n}^{r v}\right)$ and $u^{r v}=$ $k_{\text {off }}^{r v} / v^{r v}$. Inserting these solutions in Eq. (4) one obtains

$$
\begin{aligned}
p_{10}^{r v}(t+\Delta t)= & u^{r v}\left(1-e^{\left[-v^{r v} \Delta t\right]}\right), \text { if regulatory site is } \\
& \text { bound at timet } \\
p_{11}^{r v}(t+\Delta t)= & u^{r v}\left(1-e^{\left[-v^{r v} \Delta t\right]}\right)+e^{\left[-v^{r v} \Delta t\right]}, \text { if regulatory } \\
& \text { site is unbound at time } t
\end{aligned}
$$

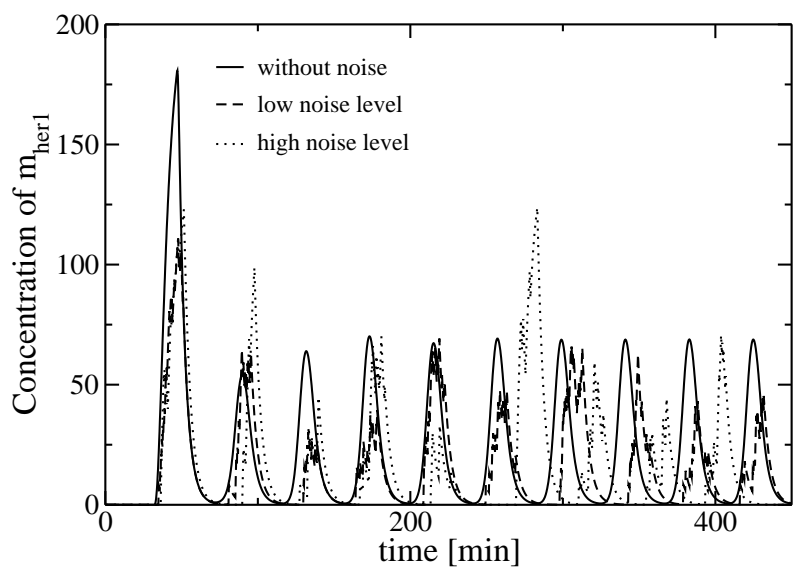

FIG. 4: Concentration of messager RNA molecules of gene her 1 as a function of time for any cell of the linear array. Comparison of results obtained using the deterministic one dimensional model described in section I (solid line) and the stochastic model for a low noise level (dashed line, $k_{o f f}^{\eta_{\text {her } 1 / 7}^{(H)}}=k_{o f f}^{\eta_{\text {her } 1 / 7}^{(D)}}=10$ and $\left.k_{\text {off }}^{\xi_{\text {deltaC }}^{(H)}}=1\right)$ and for a high noise level (dotted line, $k_{\text {off }}^{\eta_{\text {her } 1 / 7}^{(H)}}=k_{\text {off }}^{\eta_{h e r 1 / 7}^{(D)}}=1$ and $k_{\text {off }}^{\xi_{\text {delaC }}^{(H)}}=.1$ ). The parameter values are the same that in Fig. 2] The concentrations of protein and message molecules have the same behavior as the concentration of $m_{\text {her } 1}$ shown in this figure.

In the same way, the probability that any random variable $(r v)$ takes value 0 at the time $t+\Delta t$ is given by

$$
\begin{aligned}
p_{01}^{r v}(t+\Delta t)= & \left(1-u^{r v}\right)\left(1-e^{\left[-v^{r v} \Delta t\right]}\right), \text { if regulatory } \\
& \text { site is unbound at time } t \\
p_{00}^{r v}(t+\Delta t)= & 1-u^{r v}\left(1-e^{\left[-v^{r v} \Delta t\right]}\right), \text { if regulatory } \\
& \text { site is bound at time } t
\end{aligned}
$$

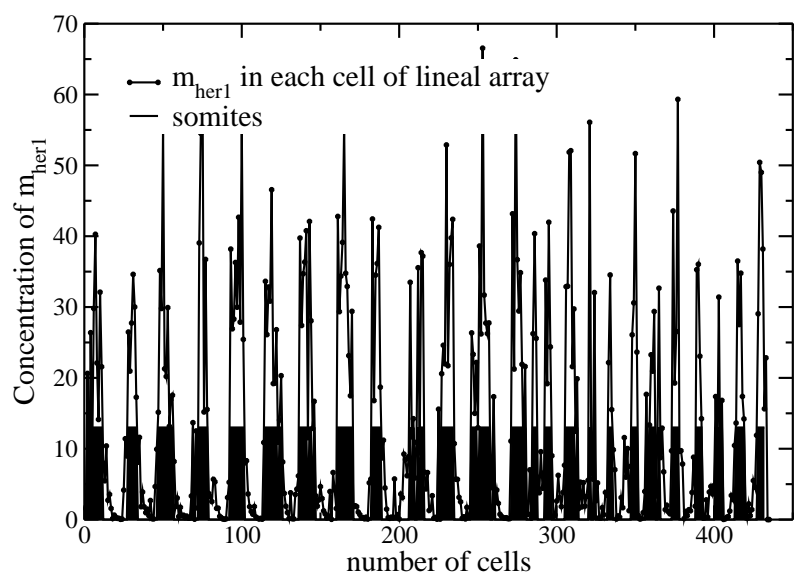

(6) FIG. 5: Concentration of message molecules of gene her 1 as a function of the number of cell at the linear array. The concentrations were calculated using the stochastic model for haploid cells with a noise level given by $k_{\text {off }}^{\eta_{h e r 1 / 7}^{(H)}}=k_{o f f}^{\eta_{\text {her1/7 }}^{(D)}}=$ 5 and $k_{\text {off }}^{\xi_{\text {deltaC }}^{(H)}}=.5$, and assuming the rest of the parameters equal to the used ones in the Fig. 2 The wavefront advances at a speed of 2 minutes by cell and 26 somites are formed at $t=900 \mathrm{~min}$, in the simulation. All the other concentrations of protein and message molecules in the cells (i.e. $p_{k}$ with $k=$ her 1 , her $7, \operatorname{deltaC}$ and $m_{k}$ with $k=$ her $7, \operatorname{deltaC}$ ) have similar behavior as the concentration of $m_{h e r 1}$.

Expressions (6) and (7) joined with the Eqs. (11) and (3) allow to calculate the concentration of protein and mRNA molecules at anytime instant for the linear array of cells considering transcription as a stochastic process. There is a correspondence between this system with noise and the deterministic system: statistical averages of the random variables $\xi_{k}^{(D)}, \xi_{k}^{(H)}, \eta_{k}^{(H)}$ and $\eta_{k}^{(D)}$ in the limit of large $k_{o f f}^{r v}$ and $k_{o n}^{r v}$ tend toward the behavior of the deterministic system. In Fig. 4, the concentration of $m_{h e r 1}$ versus time calculated using the deterministic model (detailed in the section (I) and the model with noise for two different levels of noise are compared. A random variability in the amplitude and shape of individual oscillation peaks can be seen for both results obtained with noise. In addition, as the amplitude of noise increases the oscillations of $m_{h e r 1}$ are not in phase with the deterministic model oscillations.

Finally, a somite pattern for the stochastic model is shown in Fig. 5. The wavefront advances at a speed of 2 minutes per cell freezing the oscillations of protein and mRNA concentrations and the simulation finishes at time $\mathrm{t}=900$ minutes. Thus, a pattern of 26 somites is obtained with the same constant values specified in the caption of Fig. 22 and also used in Fig. 3, It is possible to increase the number of somites decreasing the delays within the range of allowed values (see [1]). 


\section{PERTURBATION OF THE SOMITOGENESIS WAVEFRONT}

The concentration of Fgf8 signalling is high in the caudal PSM and drops between the intermediate and the rostral PSM as indicated by the top part of the Fig. 1. In this paper we assume that somite formation can begin when the Fgf8 signalling falls below a critical level, and this level moves towards the caudal end of the PSM with a constant velocity, the somitogenesis wavefront $v_{\text {wave }}$. Note we assume this velocity is not the growth rate of the PSM but in fact is faster $v_{\text {wave }}>v_{\text {growth }}$, we shall, however, assume the same velocity in both the deterministic and stochastic models. Now it is known that a transient manipulation of the wavefront in zebrafish embryos alters the size of the somites [10, 11]: larger somites result when there is transient inhibition of Fgf8 signalling, whereas smaller somites result in the presence of transient activation. Chemical inhibitor and transplantation of Fgf8 beads are used for altering the level of Fgf8 signalling that regulates the position of the wavefront within the PSM 11, 12].

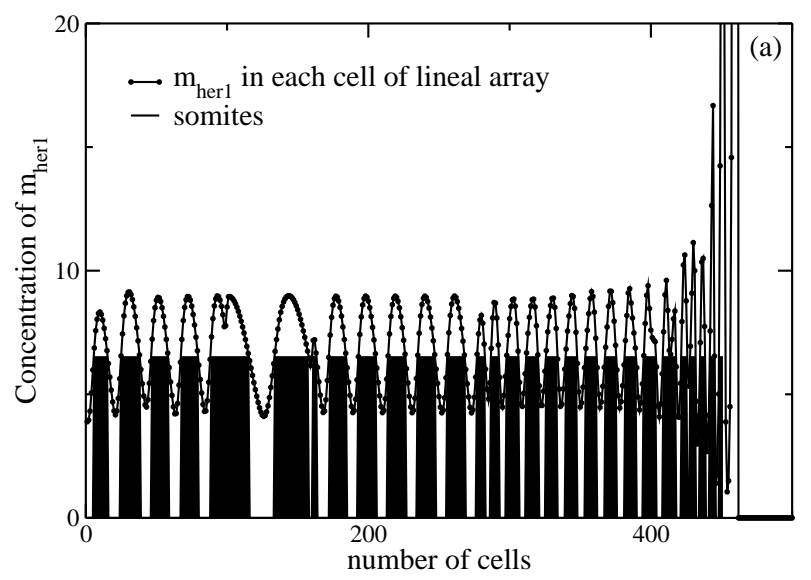

Assuming that such perturbations in Fgf8 concentration directly result in changes in the wavefront velocity, we will modify the wavefront velocity in our model and analyze their consequences. The perturbed pattern of the resulting somites depend on how the wavefront velocity $v_{\text {wave }}$ changes. In Figure 6 examples of such perturbations are shown that have been obtained with the deterministic one dimensional model by altering the wavefront velocity (Fig. 6). In each case the other parameters in the model required for finding the protein and mRNA concentrations are the same as in Fig. 3. Specifically as in the unperturbed system, the wavefront initially advances a rate of $2 \mathrm{~min}$. per cell but between the cells 100 and 160 the wavefront is perturbed. The velocity is increased to 1 min per cell in the cell interval $(100,160)$ and larger somites are formed (see Fig. 6(a)) in comparison with the results of Fig. 3. When the velocity is decreased to 4 min per cell in the same interval of cells smaller somites are formed (Fig. 6(b)). In general al-

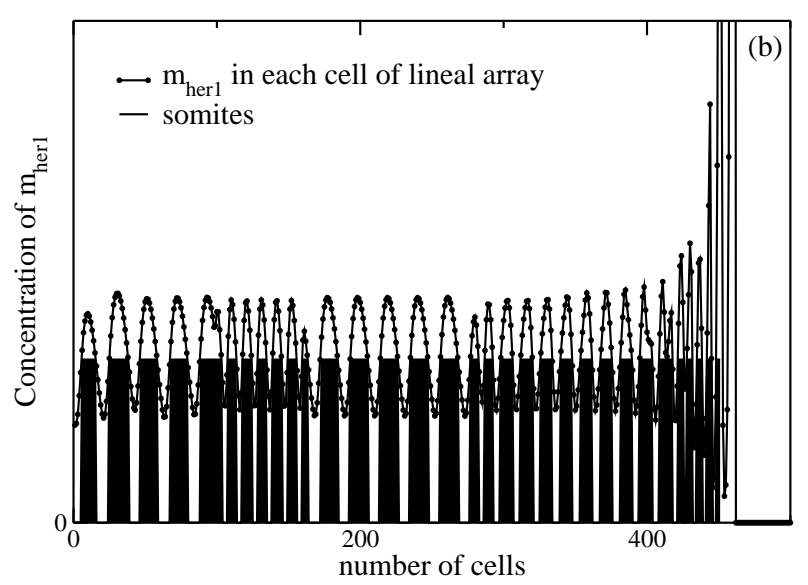

FIG. 6: Concentration of gene her 1 mRNA as a function of the number of cell in the linear array. The concentrations were calculated using the deterministic version of the one dimensional model and assuming the same parameter values as in the Fig. 2 (see caption) and 3 The wavefront advances at velocity of 2 min per cell in both figures, but between the cells 100 and 160 there is a perturbation in the Fgf8 levels resulting in a change in the wavefront velocity: the velocity decreases to $1 \mathrm{~min}$ per cell in (a) and increase to 4 min per cell in b). The patterns of somites are altered by the perturbations (see Fig. 3).

terations of the wavefront velocity perturb the resulting pattern somite in a grossly irregular manner.

\section{CONCLUSIONS}

We have carried out simulations (see Figure 5) using the deterministic version of our model that result in a growing approximately spatially periodic sequence of somites by combining the clock and wavefront model with the temporal oscillation of gene transcription and translation suggested by Lewis [1] for the zebrafish embryo (Fig. 3). We also simulated the transient manipulation of the wavefront in zebrafish embryos [10, 11]. Larger somites are formed when the wavefront velocity is locally increased whereas smaller somites results if there is a local decrease in the wavefront velocity (Fig. 6). Indeed, we believe that these are the first simulations that show in a biologically plausible manner the local variation of somite size due to external perturbation of the Fgf8 concentration.

We also noted in section [II] the consequences that gene regulation is in reality a noisy process which is likely to be crucial in the real developmental situation because of the small number of intracellular molecules involved. Our simulations also raise a number of questions for the biology os somitogenesis. First, as gene regulation is noisy it is important to study the 
consequences of this noise for somite patterning. Second, the interplay between growth at the caudal end of the PSM and the velocity of the somite development at the rostral end is crucial for the number, size and shape of the resulting somites. It would be very useful there to have more biological information of the relationship between these processes. Finally, we assume that the Fgf8 concentration acts as a morphogen creating an intracellular signalling cascade that ultimately controls transcription and translation of the gene products.
We do not have detailed information of this process and simply assume that the Fgf8 acts as an on/off switch. It is certainly possible, however, that a more complex dynamics may be involved and it would be useful to have more information on Fgf8 signalling in this context. In conclusion, further developments in the model will depend on knowledge of this biology and on the pertinence to somite formation and differention on the phase of the oscillation cycle [3].
[1] Lewis, J. (2003). Autoinhibition with Transcriptional Daley: A Simple Mechanism for Zebrafish Somitogenesis Oscillator, Current Biol. 13, 1398-1408.

[2] Pourquié, O. (2003). The segmentation Clock: Converting Embryonic Time into Spatial Pattern, Science 301, 328-330.

[3] Dale, K.J., Pourquié, O. (2000). A clock-work somite, BioEssays 22, 72-83.

[4] Baker, R.E., Schnell, S., Maini, P.K. (2006). A clock and wavefront mechnism for somite formation, Dev Biol. 293, 116-126.

[5] Aulhela, A., Pourquié, O. (2006). On periodicity and directionality of somitogenesis, Anat Embryol Rev. DOI 10.1007/s0029-006-0124-y.

[6] Pourquié, O. (2003). Vertebrate somiogenesis: a novel paradigm for animal segmentation?, Int. J. Dev. Biol. 47, 597-603.

[7] Aulhela, A., Herrmann, B.G. (2004). Segmentation in vertebrates: clock and gradient finally joined Genes and Dev. 18, 2060-2067.

[8] Palmeirim, I., Henrique, D., Ish-Horowicz, D., Pourquie, O. (1997). Avian hairy gene expression identifies a molecular clock linked to vertebrate segmentation and somitogenesis, Cell 91, 639-648.

[9] Rida, P.C.G.,Minh, N.L., Jiang, Y. (2004). A Notch feeling of somite segmentation and beyond, Dev. Biol. 265, $2-22$.
[10] Holley, S.A., Takeda, H. (2002). Catching a wave: the oscillator and wavefront that create the zebrafish somite seminars, Cell \& Dev Biol. 13, 481-488.

[11] Dubrulle, J., McGrew, M.J., Pourquié, O. (2001). FGF signalling controls somite boundary position and regulates segmentation clock control of spatiotemporal Hox gene activation, Cell 106, 219-232.

[12] Sawada, A., Shinya, M., Jiang, Y.J., Kawakami, A., Kuroiwa, A., Takeda, H. (2001). Fgf8/MARK signalling is a crucial positional cue in somite boundary formation Development 128, 4873-4880.

[13] Oates, A.C., and Ho, R.K. (2002). Hairy/E(spl)-related (Her) genes are central components of the segmentation oscillator and display redundancy with Delta/Notch signaling pathway in the formation of anterior segmental bounderies in the zebrafish, Development 129, 29292946.

[14] Henry, M.K., Urban, K.K., Dill, J.P., Merlie, M.F., Page, Kimmel C.B., and Amacher, S.L. (2002). Two linked hairy/Enhancer of split-related zebrafish genes, her1 and her7, function together to refine alternating somite boundaries, Development 129, 3693-3704.

[15] Swain, P.S., Elowitz, M.B., and Siggia, E.D. (2002). Intrinsic and extrinsic contributions to stochasticity in gene expression, Proc. Natl. Acad. Sci. USA 99, 12795-12800. 